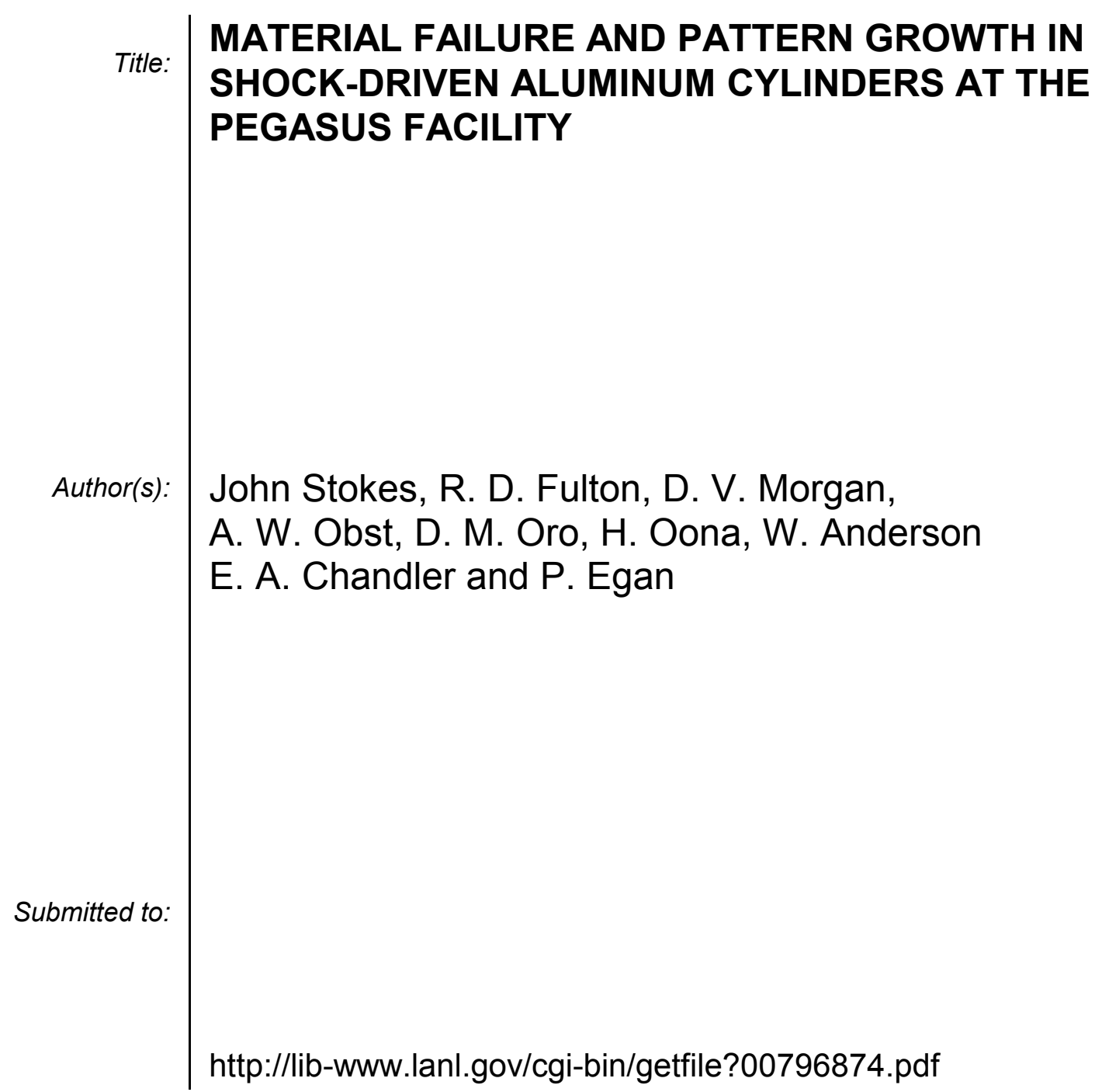




\title{
MATERIAL FAILURE AND PATTERN GROWTH IN SHOCK-DRIVEN ALUMINUM CYLINDERS AT THE PEGASUS FACILITY
}

\author{
John Stokes, R. D. Fulton, D. V. Morgan, \\ A. W. Obst, D. M. Oro, H. Oona, W. Anderson \\ Los Alamos National Laboratory, Los Alamos, New Mexico, USA \\ and \\ E. A. Chandler, P. Egan \\ Lawrence Livermore National Laboratory, Livermore, California 94551, USA
}

\begin{abstract}
Experiments on the Pegasus pulsed power facility have investigated material failure and the growth of sinusoidal perturbations machined on the free inner surface of both Al 1100 and Al 6061-T6 samples undergoing shocked acceleration. The material behind the free surface exhibits massive microspall resulting in a volume of low-density material. Rapid pattern growth in the failed material and subsequent pattern growth on the surface, including jetting in some cases, were seen. Shock pressures were $15 \mathrm{GPa}$ and $50 \mathrm{GPa}$.
\end{abstract}

\section{INTRODUCTION}

Pegasus is a $4.2 \mathrm{MJ}$ pulsed power machine used to implode a cylindrical liner $24 \mathrm{~mm}$ in radius and $0.4 \mathrm{~mm}$ thick in our experiments. The driving force is produced by the current flowing in the liner interacting with the magnetic field produced by the current. The pressure is proportional to the square of the magnetic field, which is proportional to the current times radius. We can vary this force by varying the charge on the capacitor bank.

The liner and target have been designed to produce a Taylor wave in the target to approximate the pressure wave from high explosive. This wave shape is triangular rather than a flat-toped shape which a flyer plate produces. Changing dimensions and driving current can vary the shock wave pressure profile. Pulsed power has the advantages that the grain structure effects of the explosive do not exist, and the driver is inexpensive relative to the cost of a uniform cylindrical explosive detonation. The Pegasus drive is highly symmetric, very reproducible and well diagnosed.

The diagnostic suite[1] available on Pegasus includes three to five radial and four axial x-ray images, a visible light image illuminated by a laser and/or self-emission, and various machine diagnostics [2] to measure the diving force. The radial $\mathrm{x}$-rays are recorded on film, and the axial $\mathrm{x}$ rays use a fluor with the images recorded on electronic cameras. All x-rays are independently timed. The backlighter and self-emission images are recorded on framing cameras.

Our experiments examined instabilities arising from the shock breakout in metals. Various effects, including inertial instabilities, elastic-to-plastic transition, material failure, and phase transitions contribute to the complex behavior in metals. Aluminum (Al) was chosen to avoid phase transitions at the pressures studied and to enable good radiography.

\section{DISCUSSIONS OF THE EXPERIMENTS}

The targets used in this study consisted of a cylinder shell of poly methyl methacrylate (PMMA) $2 \mathrm{~mm}$ thick, $17.5 \mathrm{~mm}$ long, with a $30 \mathrm{~mm}$ outer diameter. Lining the inside of the PMMA was an aluminum cylinder $3 \mathrm{~mm}$ thick and $17.5 \mathrm{~mm}$ long. 

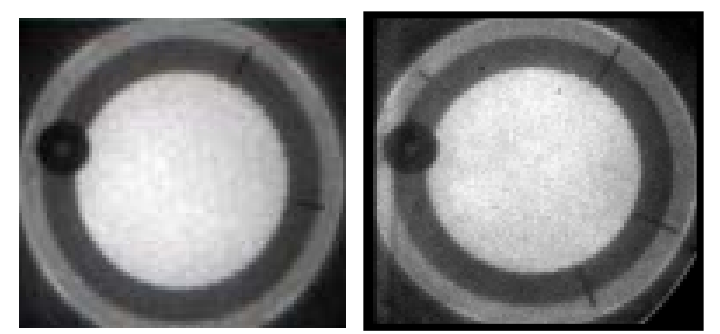

FIGURE 1 Preshot axial x-ray image of 6061 and $1100 \mathrm{Al}$ targets. The small dark circle at about $10 \mathrm{o}$ clock is a brass fitting for gas injection.

Inside the aluminum was one atmosphere of either xenon or argon gas. Endcaps were made of PMMA $12 \mathrm{~mm}$ thick. Perturbations in the azimuthal direction are machined on the inside of the aluminum. We studied the effects of shock pressure and yield strength of material on spall and on the growth of these perturbations.

One pair of experiments examined the effect of yield strength on spall and growth of instabilities. Both targets had perturbations of $8 i(1.396 \mathrm{~mm})$ wavelength and amplitudes of 0.06 and $0.12 \mathrm{~mm}$. One half of the inner circumference of each $\mathrm{Al}$ target was unperturbed and the different amplitudes were imposed on roughly $1 / 4$ of each circumference. The target on the left in Fig. 1 was 6061-T6 aluminum. Inside the aluminum was 1 atm of xenon gas. The right-hand side of Fig. 1 was $1100-\mathrm{O}$ aluminum with $1 \mathrm{~atm}$ of argon gas inside. This target had the same perturbations as the $6061 \mathrm{Al}$ plus an additional perturbation of $6 i$ wavelength and
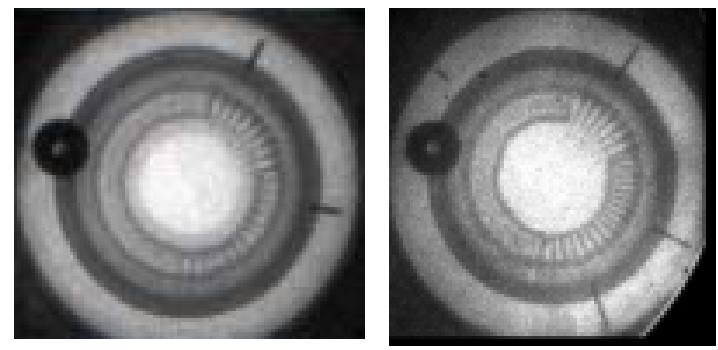

FIGURE 2. Dynamic axial x-ray images of 6061 and $1100 \mathrm{Al}$ targets at $14 \mathrm{GPa}$ shock pressure. From the center outwards the regions are unshocked gas (bright), shocked xenon (left picture only), spalled aluminum (dark ring), low-density aluminum, spalled aluminum (dark ring), low-density aluminum, normal density aluminum (outermost dark region), and PMMA. amplitude of $0.06 \mathrm{~mm}$. A $120 ;$ sector of ultra-pure aluminum was also put in the unperturbed region in the lower left-hand corner.

Figure 2 shows axial dynamic radiographs of these two experiments at about the same time. The times of the x-ray pictures are 3.38 and $3.66 \mathrm{~s}$ after liner collides with the PMMA. The shock pressure in these two experiments was $14 \mathrm{GPa}$. At this pressure, and also at $30 \mathrm{Gpa}$ in a higher pressure experiment described separately [1], the spall structures are the same and show two distinct spall layers with lower-density regions outside each. Inside the inner spall layer one can see the shocked xenon in the 6061 aluminum experiment (left). The large perturbations were unstable in both experiments, while the smaller perturbations seemed to form a crust. There appears to be no large effect due to the difference in yield strengths in the two aluminum alloys. Their yield strengths [3] are 0.04 $\mathrm{GPa}$ for 1100 aluminum and $0.29 \mathrm{GPa}$ for 6061 aluminum. However, the innermost crust of the $1100 \mathrm{Al}$ appears to be slightly thinner than the innermost crust of the $6061 \mathrm{Al}$.

The primary purpose of the experiment was to see whether a yield strength 7 to 8 times smaller, as in AL 1100-O, would affect the stability of the perturbations. Yield strength plays a significant role in determining the growth of interfacial instabilities, such as Richtmeyer-Meshkov and Rayleigh-Taylor instabilities.

An issue that has been the subject of experiments in other geometries is the effect of grain size and impurities on spall strength [4]. To test the sensitivity of the microspall pattern to grain size we substituted a segment of $99.99 \% \mathrm{Al}$. This $\mathrm{Al}$ was in the $120_{i}$ sector in the lower left corner of the right side of Figs. 1 and 2. This ultra-pure Al has larger grain sizes and fewer precipitates than the 1100 aluminum. The grains in the ultra-pure $\mathrm{Al}$ are millimeters longitudinally by $0.5 \mathrm{~mm}$ or more in cross-sectional diameter. Spall is thought to begin at void nucleation sites at grain boundaries and precipitates, and hence a difference might be expected in the spall structure. Instead we see that the spall in the region of ultra-pure $\mathrm{Al}$ has the same characteristics as the $1100 \mathrm{Al}$ region. Figure 3 shows that more deviations from "circular" were observed later in time. This may reflect morphology, such as larger pieces of spall. 

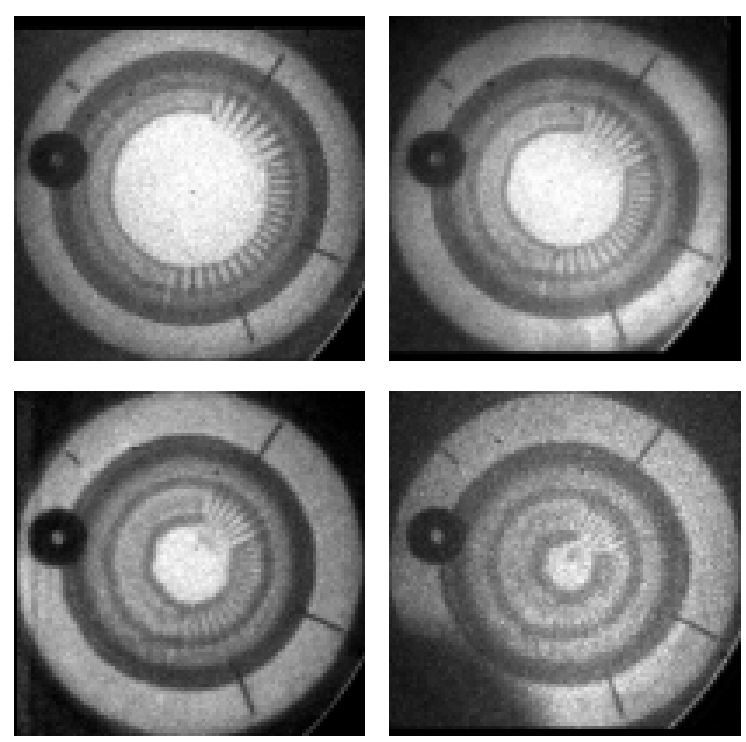

FIGURE 3. Dynamic axial $\mathrm{x}$-ray images of the $1100 \mathrm{Al}$ target with $99.99 \%$ Al 120 i sector from the marker at $10: 30$ to $6: 30$ o clock. Images were at 2.64 (upper left), 3.66 (upper right), 4.63 (lower left), and 5.37 sec (lower right) after liner impact.

Figure 4 shows a radial x-ray view of the $6061 \mathrm{Al}$ experiment shown axially on the left side of Fig. 2, and it is at the same time. We see the same microspall structure as seen axially. From the center to the left in fig. 4 we have unshocked xenon, shocked xenon, a spalled layer of $\mathrm{Al}$, a lower density region, a second spalled layer of $\mathrm{Al}$, another lower density region, the remaining aluminum, the

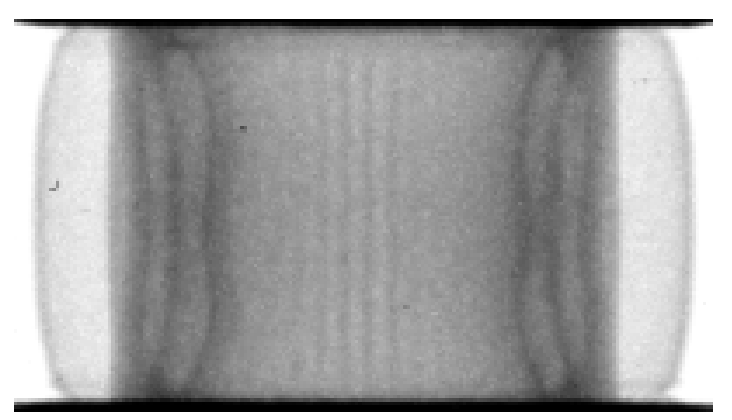

FIGURE 4. Dynamic radial $x$-ray images of the $6061 \mathrm{Al}$ target shown in Fig. 2 left side. From the center to left the regions are unshocked gas, shocked xenon, spalled aluminum (dark line), low-density aluminum, spalled aluminum (dark line), lowdensity aluminum, normal density aluminum, PMMA, and liner.

PMMA, and the liner. The center vertical stripes are the results of looking at the large amplitude perturbations machined into the aluminum. From this view we also see the end effects that we look through when we view the axial x-ray pictures. Note also that the outer $\mathrm{Al}$ is very straight showing that the liner was straight at impact. The bowing of the liner on the outside is due to end effects after impact.

A third experiment used 6061-T6 aluminum with the same $8 i$-wavelength, $\quad 0.06$-mm-amplitude perturbation as in the other 6061 aluminum target. A 24 -wavelength perturbation with $0.06 \mathrm{~mm}$ amplitude replaced the large amplitude perturbation region of the target in Fig. 2. The gas on the inside was changed to argon at $1 \mathrm{~atm}$ for better contrast in the $\mathrm{x}$-ray images. The shock pressure was increased to $50 \mathrm{GPa}$. Figure 5 shows that the $8 i$ wavelength that was stable at $14 \mathrm{Gpa}$ became unstable at 50 GPa. The growth of the $24 i$-wavelength perturbation can easily be seen. Also notice that the character of the spalled layer in the unperturbed region is different. There are two distinct density regions of spall but no thin dense spalled layers, as were observed at 14 and $30 \mathrm{Gpa}$ shock pressures.
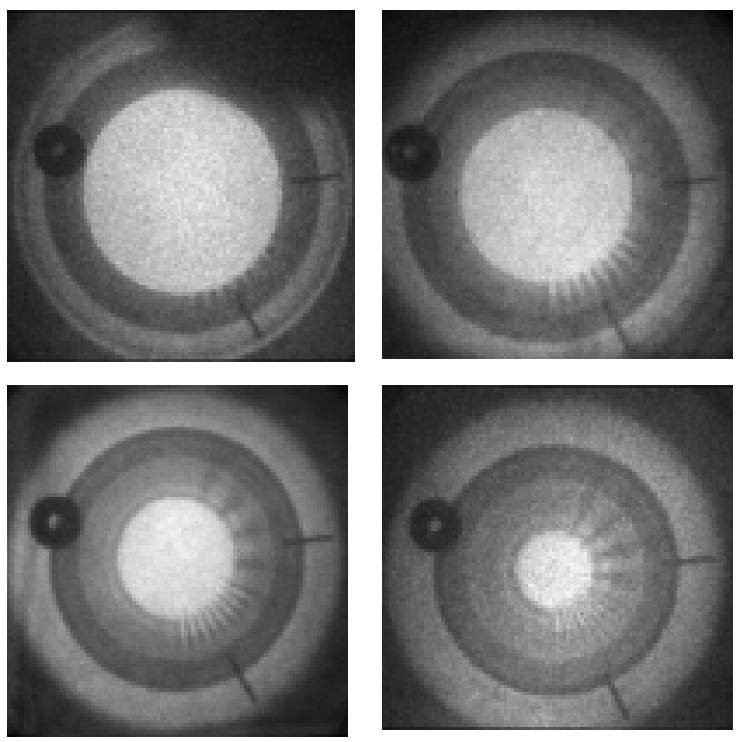

Figure 5. Dynamic axial $x$-ray images of $6061 \mathrm{Al}$ target at 50 Gpa shock pressure. The images were at $0.90,1.46,2.07$, and $2.68 \mathrm{sec}$ after liner impact. 


\section{SUMMARY}

We have observed microspallation in three types of aluminum at pressures of 14-50 GPa. The general character of the spallation is independent of the yield strength of the aluminum alloys. At pressures of 14 and $30 \mathrm{GPa}$ the spallation exhibited two spalled layers with lower density regions outside. When the pressure was increased to $50 \mathrm{GPa}$, the spall produced two different but uniform density regions. Evidence of a non-uniform spalled layer was seen in the time dependence of the ultra-pure aluminum spalled layer.

We have also studied the growth of small amplitude perturbations in both 1100 and 6061 aluminum at $14 \mathrm{GPa}$. The growth of these is also independent of yield strength. We also showed that the strength of the shock affects the stability of perturbation.

\section{ACKNOWLEDGEMENTS}

This work was performed under US DOE Contracts W-7405-ENG-48 and W-7405-ENG-36.

\section{REFERENCES}

1. Chandler, E. A., et al., Use of Pegasus Z-Pinch Machine to Study Inertial Instabilities in Al: A Preliminary Report, in Proc. of the $6^{\text {th }}$ International Workshop on the Physics of Compressible Turbulent Mixing, edited by G. Jourdan and L. Houas, Marseille, Impremerie Caractere, 1997, p. 111-115

2. Stokes, J. L., et al., Precision Current Measurements on Pegasus II Using Faraday Rotation, in Tenth IEEE International Pulsed Power Conference (Albuquerque, NM), edited by W. Baker and G. Cooperstein, IEEE, Piscataway, NJ, 1995, Vol. 1, pp. 378-383

3. Steinberg, D. J., Equation of State and Strength Properties of Selected Materials, Lawrence Livermore National Laboratory, Livermore, CA, Tech. Rep. UCRL-MA-106439, 1996

4. Meyers, M. A., Dynamic Behavior of Materials, Wiley and Sons, New York, 1994, pp. 541-546 